\title{
Lutz Marz \\ Implosion und Stagnovation. \\ Probleme, Phänomene und Pfade moderner \\ Modernisierung
}

Zusammenfassung: In der DDR ist eine jahrzehntealte Organisationsweise des gesellschaftlichen Lebensprozesses - der "real existierende Sozialismus - in wenigen Monaten implodiert. Ein Teil der Wissenschaft reagiert auf dieses Phänomen mit einem doppelten Schweigen. Erklärungsbedürfnis und Erklärungsmacht scheinen hier in einem eigentümlichen Verhältnis zueinander zu stehen. Gerade wegen dieses Schweigens wäre zu fragen, ob, und wenn ja in welcher Hinsicht, dieser ebenso unerwartete wie rasante Implosionsprozeß einer prämodernen Gesellschaftsordnung auch die Modernisierung moderner Gesellschaften betreffen könnte.

\section{Das Problem: Die Implosion der prämodernen Alternative}

Freilich, das Reizwort »real existierender Sozialismus « ist eine ideologische Kategorie. Wie jene fiktiven Vokabeln, durch die er definiert wurde - so etwa »Volkseigentum « oder »Planwirtschaft « (Marz 1990a, S. 21) -, erzeugte auch und gerade dieser Begriff permanent eine Scheinwelt: In dem Bild, das er suggerierte war die Realität - die spezifische Organisationsweise des gesellschaftlichen Lebensprozesses - die er zu bezeichnen vorgab, verdreht und auf den Kopf gestellt. Dennoch sollte man sich nicht allzuschnell von ihm verabschieden, denn trotz dieser eklatanten Differenz von Begriff und Wirklichkeit, die ja weder dem alltagsweltlichen Verstand noch der sozialwissenschaftlichen Vernunft verborgen blieb, übte dieses Kultwort, wie vielleicht kein anderes, eine geradezu magische Faszination aus - und die nicht nur auf diejenigen, die in dieser Welt lebten und sie gestalteten, sondern auch auf jene, die sie mit Sympathie beobachteten, ihr kritisch und/oder gar feindlich gegenüberstanden. Dieses Faszinationsphänomen läßt sich nur sehr bedingt mit dem bloßen Verweis auf die penetrante Agitation und Propaganda des Parteiapparats erklären. So flach nämlich das Codewort »real existierender Sozialismus « aus sozialwissenschaftlicher Forschungsperspektive auf den ersten Blick auch erscheinen mochte, so funktionierte es doch als ein exklusiver denk- und handlungsleitender Meß- und Orientierungspunkt, von dem aus sich die Entwicklungsdynamik der komplexen Weltgesellschaft klar überblicken und mit einer doppelten Dual-Codierung über zwei weltanschauliche Leitdifferenzen verblüffend einfach wie folgt kartographieren ließ:

Erstens wurde die wirkliche Welt aller existierenden Gesellschaftsformen über das digitale Beobachtungsraster »Kapitalismus/Sozialismus « in ein zweispaltiges Tableu (»erste« und »zweite« Welt) projiziert, in dem ein Randfeld (»dritte« Welt) für all jene 
Gesellschaftsformen reserviert war, die sich diesen beiden Spalten noch nicht eindeutig zuordnen ließen, die jedoch früher oder später durch die objektiven Gesetzmäßigkeiten - insbesondere die Logik des »Privateigentums « bzw. die Logik des »Volkseigentums « - zwangsläufig auf einen dieser beiden menschheitsgeschichtlichen Entwicklungswege getrieben wurden. Da nun der kapitalistische Entwicklungspfad als eine sich tendenziell verengende Sackgasse, der sozialistische hingegen als ein sich stetig öffnendes Feld vorgestellt wurden, konnte jeder aktuelle Systemvergleich noch so deprimierend ausfallen, potentiell waren die »sozialistischen« den »kapitalistischen « Gesellschaften notwendig überlegen. Wenn nämlich auch der Sozialismus dem Kapitalismus auf vielen vielleicht sogar fast allen Gebieten hinterherhinkte, ja selbst wenn sich der Abstand vergrößerte, war dies kein Grund zu substanziellen Irritationen, denn wieweit auch immer diese durch die Geschichte objektiv zum Tode verurteilte Gesellschaftsformation tatsächlich oder vermeintlich dem Sozialismus voraus sein mochte, dieses » voraus « lag per definitionem immer in der Sackgasse.

Zweitens wurde die mögliche Welt aller denkbaren Gesellschaftsformen über das digitale Bewertungsraster »real/irreal « in ein zweizeiliges Tableau projiziert (»praktizierte « und »illusionäre « Gesellschaftskonzeptionen), wobei ein Randfeld (Reformstrategien «) jenen Gesellschaftskonzepten vorbehalten blieb, die nur die Oberfläche oder singuläre Gebiete der Gesellschaft verändern, die Kernstrukturen hingegen unberührt lassen wollten, die aber auf ihren verschiedenen alltragspraktischen Wegen über kurz oder lang unweigerlich auf jenen alternativen Verzweigungspunkt stoßen mußten, wo sie gezwungen waren, sich entweder illusionär zu verflachen oder fundamental zu radikalisieren.

Im Begriff »real existierender Sozialismus « schoben sich diese beiden Teiltableaus in einer weltanschaulichen Matrix zusammen, die das individuelle wie das kollektive Denken und Handeln in einer Richtung fokussierte, denn da »real existierend « handfeste Praxisorientierung und nachprüfbare Wirklichkeitsbezogenheit von wilder Spekulation und wüster Phantasterei, »Sozialismus « permanente Zukunftsoffenheit und wachsende Entwicklungschancen von zunehmender Perspektivlosigkeit und kumulierenden Instabilitäten schied, gab es innerhalb dieser Matrix nur vier denkbare Geselischaftsmodelle: Erstens den »real existierenden Sozialismus «, dem bei all seinen akuten Problemen potentiell die Zukunft gehörte, zweitens den »real existierenden Kapitalismus «, der sich trotz aller momentanen Überlegenheit mit kapitallogischer Konsequenz in eine weltgeschichtliche Sackgasse hineinproduzierte, drittens den »nicht real existierenden Sozialismus «, als »linke « und viertens den »nicht real existierenden Kapitalismus«, als »rechte« Illusion eines möglichen Weges jenseits der ausgetretenen »realkapitalistischen « oder »realsozialistischen « Entwicklungspfade. Alternatives gesellschaftkonzeptionelles Denken und Handeln, das festen Grund sucht, sich weder im irrealen Terrain »linker« oder »rechter Phantasien verirren, noch in der realen Sackgasse kapitalistischer Entwicklungsdynamik verlieren wollte, wurde so zwangsläufig auf den »real existierenden Sozialismus « verwiesen. Er war nicht nur der imaginäre Fluchtpunkt aller antikapitalistischen Alternativen und der 
virtuelle Schnittpunkt aller konkretsozialistischen Utopien, sondern auch der permanente Reibungspunkt aller nicht- oder gar antisozialistischen Gesellschaftsprogrammatik.

Natürlich lagen all diese Punkte nicht auf der Oberfläche dieser Gesellschaft, sondern in jenen vermeintlichen inneren Werten und strukturellen Potenzen, von denen nicht nur die Propaganda so geschwätzig redete, sondern die viele selbst hinter der rohen Gestalt dieses Gesellschaftskörpers glaubten aufscheinen zu sehen. Wie eine Fata Morgana schwebte ïber den verwüsteten Alltagspraxen der Länder des »real existierenden Sozialismus « die Vorstellung, der »eigentliche « Kern, das »tatsächliche« Wesen und der »wirkliche « Inhalt dieses Typs der Gesellschaftsorganisation würden irgendwann in einem von einer reformierten Partei geführten und von den Volksmassen getragenen Prozeß die schlechte Schale, die schmutzige Erscheinung und die negativen Formen endgültig aufsprengen. Egal ob man dies erhoffte oder befürchtete, ob man nur beobachtete oder auch teilnahm, ob man diesen Prozeß in Gang setzen oder verhindern wollte, direkt und indirekt, gewollt und nicht gewollt, bewußt und unbewußt, wurde diese Vorstellung wieder und wieder (re-)produziert - ob in byzantinischen Jubelfeiern, solidarischen Kritiken, markigen Attacken, feinsinnigen Kunstwerken oder distanzierter Teilnahmslosigkeit. Die verschiedensten Diskurse ließen - auch in ihren beredten Leerzeichen - zwei scheinbare Evidenzen auskristallisieren:

Erstens, es existiert eine anschau- und anfaßbare Alternative jenseits und gegenüber postmoderner Gesellschaftsentwicklung und, zweitens, von dieser Alternative strahlt - aktuell und/oder potentiell - ein Transformationsdruck auf die Kernstrukturen bürgerlichen Gesellschaften aus. Wie immer man sich gegenüber diesen beiden Evidenzen auch konkret theoretisch, ideologisch oder politisch verorten mochte, man bezog sich auf sie bzw. wurde auf sie bezogen. Nahezu alles schien möglich - daß sich diese Alternative schrittweise oder explosiv reformiert, daß sie ewig stagniert oder langsam dahinsiecht -, nur eins lag außerhalb des Vorstellungshorizontes - daß sie mit einem so atemberaubenden Tempo implodiert. Noch vor einem Jahr schien es undenkbar, daß ein Land des »real existierenden Sozialismus « innerhalb weniger Monate zusammenbrechen könnte.

Eben dies Unvorstellbare ist in der DDR geschehen: die Implosion der welthistorischen Alternative. Das vor kurzem noch Undenkbare ist zur aktuellen tagespolitischen Aufgabe geworden: die Transformation der prämodernen Alternative.

Es existiert ein eskalierender Handlungsbedarf.

Existiert auch ein Erklärungsbedürfnis?

\section{Das Phänomen: Das doppelte Schweigen der Wissenschaft}

Die äußeren Daten dieses Implosionsprozesses sind zum Greifen nahe: 7.10.1989, 9.10.1989, 4.11.1989, 9.11.1989, 31.12.1989, 2.1.1990, 15.1.1990, 18.3.1990, 1.7.1990, September, Dezember, 1991... Begriffen ist er noch längst nicht. Die Welt 
hat sich nachdrücklich verändert. Allein, die Interpretation dieser Veränderung stimmt nachdenklich: Auf den Wandel der Wirklichkeit reagiert ein Teil der Wissenschaft mit doppeltem Schweigen.

\section{Das explizite Schweigen: Die äußere Leerstelle des Diskurses}

Verfolgt man das diskursive Netz; das sich um den Implosionsprozeß der Alternative spannt, ist eine bestimmte Asymmetrie nur schwer zu übersehen. An einigen Stellen ist es geradezu filigran geknüpft, an anderen doch recht großmaschig ausgelegt. Vieles spricht dafür, daß diese Leerstellen zwischen den Diskurslinien nicht zufällig auftreten, sondern eher aus dem eigentümlichen Verhältnis resultieren, in dem Erklärungsbedürfnis und Erklärungsmacht vielfach zueinander stehen: Wo ein Erklärungsbedürfnis existiert, fehlt oft die Erklärungsmacht, wo die Erklärungsmacht vorhanden zu sein scheint, fehlt nicht selten das Erklärungsbedürfnis. Die einen wollen diesen Prozeß erklären, können es jedoch nicht, die anderen scheinen ihn erklären zu können, wollen es jedoch nicht.

Es kann schwerlich erstaunen, daß sich in der DDR ein großes, oft genug auch ein geradezu existenzielles Erklärungsbedürfnis entwickelte. Die zunehmende Implosion der Alternative erzeugte zwangsläufig einen wachsenden Erklärungsdruck. Insbesondere zehntausende »M/L'er « sahen sich diesem Druck ausgesetzt. Sie, die zuvor landauf, landab - an den Universitäten und Akademien, den Hoch- und Fachschulen, den Bezirks-, Kreis- und Sonderschulen der SED, des FDGB und der FDJ - jahrzehntelang zu begründen hatten, warum es unmöglich zu so einem Prozeß kommen könnte, sahen sich nun vor die Aufgabe gestellt, zu erklären, warum es notwendig dazu kommen mußte. Diese Aufgabe kam einer Quadratur des Kreises gleich. Der Erklärungsdruck entzog den »M/L'ern « aus zwei Richtungen ihre Legitimation als Wissenschaftler: Wer sich ihr nicht stellte, sie schlecht oder gar nicht löste, der mußte sich fragen lassen, warum er, der sonst auf jede Frage eine Antwort wußte, zu dem, was da auf der Hand lag, nun nichts bzw. nicht Überzeugendes zu sagen hatte - das Wissen wurde in Frage gestellt. Wer jedoch meinte, die Aufgabe befriedigend oder gut gelöst zu haben, der mußte sich fragen lassen, woher denn sein plötzliches Wissen kam, warum er es nicht längst publik gemacht hatte, ja welche Rolle er vorher überhaupt spielte - das Gewissen wurde in Frage gestellt.

Doch dem Erklärungsdruck war nicht nur subjektiv sehr schwer standzuhalten, ihm war auch objektiv kaum zu entsprechen. Wer diesen plötzlichen Implosionsprozeß ob aus psychischem Selbstzwang oder sozialem Fremdzwang - erklären wollte, dem wurde nur allzuschnell sehr schmerzlich bewußt, daß er dies nicht vermochte. Dieses Unvermögen der Einzelnen war nicht zuletzt Ausdruck der allgemeinen Begriffs- und Erklärungsohnmacht, die das Forschungsterrain erzeugte, auf dem sie sich bisher angesiedelt hatten. Die Wissenschaftslandschaft insgesamt war schon merkwürdig genug. Doch konnte man in anderen (natur- oder technikwissenschaftlichen) Forschungsfeldern vielleicht noch von einer Hügellandschaft sprechen, die Ausblicke auf das internationale Wissenschaftsterrain ermöglichte, stieß man in der Sozialwis- 
senschaft auf ein geradezu skuriles Profil (Engler 1990). Institutioneller Granit, geistige Steppe und diskursive Wüste, soweit das Auge reichte - nur ab und zu einzelne Wissenschaftler, die sich auf das internationale Niveau sozialwissenschaftlicher Forschung heraufgearbeitet hatten, es nicht nur überblickten, sondern von Fall zu Fall auch überragten. Als nun die Dämme brachen und sich der Strom sozialwissenschaftlichen Wissens ungehindert in diese merkwürdige Landschaft wälzen konnte, wurde er zunächst begierig aufgesogen. Doch bald stand vielen das Wasser bis zum Hals. Vergeblich suchte man sich auf tatsächliche oder vermeintliche konzeptionelle Erhebungen zuriickzuziehen. Für nicht wenige war es bereits zu spät. Wo man sich vorher jahrelang in der Sonne des wahren Wissens ebenso ausgeruht wie ausgedörrt hatte, da konnte man nun diesen Strom nicht auf ein Mal schlucken, geschweige denn mit eigenen Gedanken bändigen. Die öde Landschaft wurde zum Sumpf.

Im Gegensatz dazu scheinen. Erklärungsbedürfnis und Erklärungsmacht im westlichen Wissenschaftsbetrieb in einem umgekehrten Verhältnis zueinander zu stehen. Das Problem besteht hier vielleicht nicht so sehr darin, daß man das Gefühl hat, nicht erklären zu können, als vielmehr darin, daß man stillschweigend in der Annahme übereinzustimmen scheint, nichts erklären zu brauchen. Wenn jemand überhaupt ein strategisches Forschungsinteresse am Zusammenbruch des »real existierenden Sozialismus « in der DDR entwickeln könnte, dann die Historiker. So sehr der rasante Implosionsprozeß viele Sozialwissenschaftler ideologisch, politisch oder persönlich berührt hat, so wenig tangierte er ihre unmittelbaren Forschungsinteressen. Der Vorgang scheint das sozialwissenschaftliche Wissensgebäude offensichtlich eher stabilisiert, denn erschüttert zu haben. Der Kollaps einer prämodernen Gesellschaft irritiert die Kernstrukturen des postmodemen Problembewußtseins kaum. Es sieht sich mehr bestätigt als verunsichert (Friese/Wagner 1990). Sicher, eine gewisses Forensik dieser monumentalen Sozialpathologie mag eine Zeitlang noch vonnöten sein, doch die großen, neuen und eigentlichen Problemfelder liegen vorn, nicht hinten. Die Frage, ob im Tod des welthistorischen Gestern nicht auch Probleme der weltgesellschaftlichen Zukunft aufscheinen, die es wert wären in das perspektivische Blickfeld der Wissenschaft gerückt zu werden, wird selten genug gestellt. Wo es das Morgen zu bewältigen gilt, da kann man sich nicht gedanklich im Vorgestern abarbeiten. Man hat die Erklärungsmacht, aber die Tatsachen haben gesprochen, deutlich genug, warum sollte man dem noch viel hinzufügen.

Hat man die Erklärungsmacht tatsächlich?

\section{Das implizite Schweigen: Die innere Leerstelle des Diskurses}

Betrachtet man das diskursive Netz, das sich um den Implosionsprozeß spannt mit einigem reflexiven Abstand, dann fällt auf, daß große Teile davon in einer Art »Erklärungskubus « liegen, der durch drei »W-Achsen« gebildet wird. Dieser Kubus ließe sich, sehr vereinfacht, wie folgt skizzieren:

Erstens, die drei »W-Achsen«: Die »Wer-Achse« definiert, wer im gesellschaftlichen Raum Veränderungen bewirkt. Sie bestimmt die Quelle der Veränderungen. Das 
Grobraster dieser Achse unterscheidet etwa zwischen Individuen (charismatische Führer, einsame Genies, pfiffige Erfinder...), Gruppen (Eliten, unterdrückte Klassen, Avantgarden, Randgruppen, Expertenkulturen...) oder Strukturen (Interessenkonflikten zwischen Individuen oder Gruppen, gesellschaftliche Verhältnisse, Widersprüche zwischen den Verhältnissen, Subkulturen...). Die »Wo-Achse« definiert, wo die konkrete Quelle im gesellschaftlichen Raum liegt. Sie bestimmt den Ort der Veränderung. Das Grobraster dieser Achse differenziert zum Beispiel zwischen segmentären Einheiten, stratifikatorischen Ebenen (Mikro-, Meso- oder Makroebenen) oder monofunktional ausdifferenzierten Teilsystemen (Wirtschaft, Wissenschaft, Politik, Bildung...). Die »Wie-Achse« definiert, wie die konkrete Quelle an ihrem spezifischen Ort im gesellschaftlichen Raum die Veränderungen zustandebringt. Sie bestimmt den Modus der Veränderung. Das Grobraster dieser Achse unterscheidet insbesondere zwischen Kontextualismus und Universalismus (Engler 1989b).

Zweitens, die unendliche Verfeinerung: Die Grobraster der Achsen verleihen dem sozialwissenschaftlichen Blick Trennschärfe. Es segmentiert den zunächst diffusen Forschungsgegenstand in abgegrenzte Untersuchungsräume, die mit den verschiedenen grundlagentheoretischen und/oder empirischen Methoden arbeitsteilig erschlossen werden können. Jede äußere Abgrenzung der Forschungsräume läßt sich in dem Maße sukzessiv verfeinern, wie deren innere Erkundung voranschreitet, sich also die Tiefenschärfe des sozialwissenschaftlichen Blicks entfaltet. In jedem Forschungsraum des »Erklärungskubus « lassen sich weitere, neue Forschungsräume abstecken. Trennschärfe und Tiefenschärfe des sozialwissenschaftlichen Blicks treiben sich wechselseitig voran.

Drittens, der ewige Motor: Die permanente infinitesimale Verfeinerung auf den Achsen erzeugt immer diffizilere konzeptionelle Bausteine. Aus dieser unerschöpflichen Quelle ergießt sich ein ewiger Strom von Rohmaterial für neue Erklärungssätze. Durch eine laufende (Re)kombination dieses Materials lassen sich ständig neue Erklärungsansätze produzieren. Diese wiederum definieren Inhalt, Form und Richtung der weiteren Verfeinerung. Zwei Prozesse sind hier rekursiv geschlossen: Die Verfeinerung auf den Achsen und die Kombination zwischen den Achsen. Dieser rekursive Zusammenschluß ist der ewige Motor des »Erklärungskubus «. Er hält ihn zusammen. Der offensichtliche Vorteil dieses »Erklärungskubus « besteht darin, daß er erstens stets ein unerschöpfliches Reservoir an neuen Erklärungsansätzen bereit hält und daß sich daraus zweitens eine Vielzahl flexibler Interpretationsnetze knüpfen lassen, um damit jedes Veränderungsphänomen aus den unterschiedlichsten Richtungen kategorial einzufangen. Sein verborgener Nachteil besteht darin, daß er eine innere Kehrseite, eine systematische konkrete Leerstelle besitzt. Der ewige Motor des »Erklärungskubus setzt nämlich einen Mechanismus der Reduktion/Kombination in Gang. Zunächst wird der Forschungsgegenstand in immer kleinere Teile zerlegt, bis eins dieser isolierten Bruchstücke analysierbar wird, anschließend werden diese dann (re-)kombiniert. Das Problem besteht hierbei darin, daß das Ganze nicht nur einfach quantitativ mehr ist als die Summe seiner Teile, sondern das es auch etwas qualitativ Anderes ist. Was durch die Raster des »Erklärungskubus« fällt, ist gerade dieses 
Andere-die Komplexität, Ganzheit und Synthesis des Forschungsgegenstandes. Der ewige Motor erzeugt immer wieder die Differenz zwischen dem Entwicklungsniveau »zusammenfassender theoretischer Modelle« von sozialen Phänomenen und der Menge des »unzusammengefaßten Einzelwissens« (Elias 1985, S. 7) über diese. Aus dieser Differenz entspringt ein Synthetisierungsdruck, der darauf zielt, die »AnalyseSynthese-Balance « (Engler 1989a, S. 754) sozialwissenschatlicher Forschung besser auszurichten. Sie läßt sich nur »durch zweispännige Untersuchungen auf der theoretischen und der empirischen Ebene in engster Tuchfühlung miteinander (Elias 1985, S. 52) schließen, wobei dies jedoch nicht im Sinne eines synchronisierten hautnahen Parallellaufs zweier, letztlich doch getrennter Forschungseinrichtungen mißverstanden werden darf, geht es doch vor allem darum, daß sich beide Richtungen wechselseitig strukturieren. Hierbei ist man darauf angewiesen, mit Metaphern zu arbeiten, die dann durch ihren kollektiven Gebrauch schrittweise in sozialwissenschaftliche Fachausdrücke transformiert werden (Elias 1985, S. 349). Für gewöhnlich fällt dieser Nachteil nicht auf. Er liegt im blinden Fleck der Erklärungsmuster. So kann er sich etwa in den Leerstellen der Beschreibung eines Veränderungsprozesses verbergen: Wann ist ein solcher Prozeß schon einmal so lückenlos und präzise dokumentiert, daß alle drei Achsen in ihrer vollen Ausdehnung ins Blickfeld geraten? Oder er schlüpft unerkannt durch die Maschen der Erklärungsnetze: Wann läuft ein solcher Prozeß schon einmal so kompakt und komprimiert ab, daß das Moment des Synthetischen für jedermann blitzartig augenfällig wird und als das eigentlich erklärungsbediurftige Phänomen ins Auge springt? Schließlich mag es ihm gelingen, ins Dunkel des Vergessens zu entkommen: Wann entfaltet ein solcher Prozeß schon einmal so eine explosionsartige Dynamik, daß er - wieder und wieder, nahezu tagtäglich - eben dieses Phänomen permanent neu erzeugt? - Ja und wann träfe all dies einmal so gebündelt zusammen?

Es ist zusammengetroffen. Ein Land des $»$ real existierenden Sozialismus « ist in wenigen Monaten implodiert.

Doch warum sollte dies Faktum die Modernisierung moderner Gesellschaften problematisieren?

\section{Der Pfad: Die Problematisierung eines Modernisierungstyps}

Ein einfache Frage.

Die Antwortsuche sollte da nicht schwerfallen, bräuchte man doch nur die Experten der Wahrheit zu befragen - die Wissenschaftler. Sie, die Fachleute des Wissens, dürften doch um Antworten nicht verlegen sein. Bedauerlicherweise gibt es da einen fatalen Verdacht.

Angenommen, sie, die Experten, wären gar keine Experten? Angenommen, sie, die Experten des Wissens, wären gar nicht die einzigen, die Wahrheiten produzierten und ihre exklusive Kompetenz wäre eine ebenso sehr gesellschaftlich zugesprochene wie kollektiv eingebildete? 
Der Verdacht ist weder aus der Luft gegriffen, noch läßt er sich übersehen, denn die Experten des Wissens verdächtigen sich selbst und zeigen sich öffentlich an. Freilich, er ließe sich ohne weitere reflexive Umstände verdrängen.

\section{Der Ansatz: Die Interkommunikation der Wissens-Kulturen}

Doch vielleicht sollte man sich die Umstände machen und grundsätzlich fragen: Gibt es einen Standpunkt, vom dem aus sich die Frage nach dem Zusammenhang zwischen Implosionsproze $ß$ und Modernisierungsproze $ß$ beantworten ließe?

Erklärungen werden in Wissens-Kulturen (re-)produziert. Die Wahrheit einer Wissens-Kultur wird aus zwei Richtungen konstruiert. Erstens stellt jede Wissens-Kultur als Wissens-Kultur eine Zeichenmenge dar, deren dreifache Bedeutung - objektive Wahrheit, intersubjektive Richtigkeit und subjektiver Sinn - sich in gegenstands(Subjekt-Objekt), interaktions- (Subjekt A - Subjekt B) und subjekt- (Subjekt) bezogenen Handlungen reproduziert. Dies führt intrakulturell zur Herausbildung von drei verschiedenen argumentativen Diskurslinien, mit je spezifischen Rationalitäten. Die Wahrheit einer Wissens-Kultur definiert sich so stets jenseits, nicht selten auch gegenüber ihrer Richtigkeit und ihrem Sinn (Krüger 1990b, S. 215). Zweitens stellt jede Wissens-Kultur als Wissens-Kultur eine Zeichenmenge dar, in der sich die darin residenten drei Funktionen - Symptom (Ausdruck), Symbol (Darstellung) und Signal (Appell) - in funktionsspezifisch verstärkender/hemmender Weise verschränken: Symbol-Verstärkung/Symptom- und Signal-Hemmung (wissenschaftliches Wissen), Symptom- und Symbol-Verstärkung/Signal-Hemmung (künstliches Wissen), Symptom- und Signal-Verstärkung/Symbol-Hemmung (ideologisches Wissen) etc. Dies führt interkulturell zur Herausbildung von verschiedenen Wissens-Kulturen, mit je spezifischen Rationalitäten. Die Wahrheit einer Wissenskultur definiert sich so stets jenseits, meist vor allem auch gegenüber diesen anderen Wissens-Kulturen (Bühler 1965, S. 28; Engler 1989c, S. 344 ff.). Dies ist eine wissenschaftliche Aussage eines Wissenschaftlers über Wissenskulturen in einer wissenschaftlichen Zeitschrift, sie ist mehrfach selbstreferenziell und unterliegt damit selbstverständlich allen dementsprechenden Paradoxien.

Aus der besonderen Perspektive der wissenschaftlichen Wissens-Kultur kann es nun mindestens aus folgenden drei Gründen keine Meta-Wissens-Kultur geben:

Erstens existiert keine Wissens-Kultur, die einen exklusiven Meta-Status erlangen könnte, weil es weder eine Meta-Wissens-Kultur (Gödel 1931) noch eine Meta-Wissens-Kultur (Maturana/Varela 1987) geben kann.

Zweitens existiert keine Wissens-Kultur, die metakulturell die Reproduktion der Kooperation zwischen den Wissens-Kulturen koordinieren, mithin eine exklusive MetaKommunikation entfalten könnte (Krüger 1988; Habermas 1981, S. 571 ff.; Krüger 1989).

Drittens ist empirisch beobachtbar, daß Wissens-Kulturen, die versuchten, sich über ein spezielles Macht-Dispositiv einen Meta Status zu sichern und eine Meta-Kommunikation zu entfalten, sich mit diesem Versuch selbst vernichteten. ${ }^{4}$ 
Wenn es also aus wissenschaftlicher Sicht keine Meta-Wissens-Kultur geben kann, dann verbietet es sich die wissenschaftliche Wissens-Kultur selbst, einen diesbezüglichen Geltungsanspruch zu entwickeln - was natürlich keinesfalls bedeutet, daß ihr deshalb gesellschaftlich nicht dieser Status zugesprochen werden könnte oder daß die Mitglieder dieser Wissens-Kultur - die Wissenschaftler - sich selbst einen solchen verleihen.

Existiert kein absoluter, extramundaner, gleichsam göttlicher Standpunkt, von dem aus sich die Frage nach einem möglichen Zusammenhang zwischen der Implosion einer »real sozialistischen « und der Modernisierung einer modernen Gesellschaft allgemeingültig beantworten ließe, dann stellt sich zwangsläufig folgende Frage: Ist es überhaupt denkbar, den Zusammenhang zwischen Implosions- und Modernisierungsprozeß so zu thematisieren, daß eine möglichst viele Wissens-Kulturen einschließende Interkommunikation in Gang gesetzt wird?

Es ist denkbar, nämlich dann, wenn es gelänge, ein, die unterschiedlichen WissensKulturen übergreifendes Phänomen zu finden, das sie nicht nur tangiert, sondern existenziell betrifft und in dem sich dieser Zusammenhang aus den je verschiedenen Perspektiven zentrieren ließe.

Gibt es ein solches Phänomen?

\section{Die Frage: Stagnovation als Modernisierungstyp?}

Zweifellos gibt es viele Phänomene dieses Implosionsprozesses, die es wert wären, eingehend untersucht zu werden. Allein, ein Problem ist, mehr als alle anderen, in besonderem Maße erklärungsbedürftig. Es ist ebenso der Dreh- und Angelpunkt dieses Prozesses, wie es in all seinen Momenten aufscheint: Die Ohnmacht der Gesellschaft gegenüber ihrer Krise. Überall stößt man auf diese individuelle, kollektive und gesamtgesellschaftliche Ohnmacht. Nicht der spektakuläre Zusammenbruch der Allmacht, sondern die permanente (Re-)produktion der Ohnmacht an allen Punkten des gesellschaftlichen Raumes zeichnen den Implosionsprozeß des »real existierenden Sozialismus « aus. Nicht so sehr daß diese Gesellschaft zusammenbrach, sondern wie dies geschah, verdient Aufmerksamkeit. Die weitverbreitete konkrete Denk- und Handlungsohnmacht läßt sich nur bedingt aus der allgemeinen Übermacht der bürgerlichen Gesellschaft erklären. Der Implosionsprozeß wird nicht primär durch eine äußere Macht forciert, sondern durch die innere Ohnmacht beschleunigt. Er ist wesentlich ein Kreislauf der Ohnmacht.

Dieses Problem betrifft den Modernisierungsprozeß moderner Gesellschaften doppelt. Erstens wäre zu fragen, ob das, was sich in der DDR ereignet hat, nur ein deutsch/ deutsches Sonderphänomen darstellt oder ob sich solche Implosionsprozesse in anderen Gesellschaften des »realexistierenden Sozialismus « nicht mit ähnlicher Plötzlichkeit und Geschwindigkeit wiederholen könnten. Wäre dies denkbar, müßte weiter gefragt werden, ob die Gefahr besteht, daß diese Prozesse interferieren und in einer Art Kettenreaktion eskalieren. Wer dies umstandslos für undenkbar hält, muß daran erinnert werden, was noch alles vor einem Jahr als undenkbar galt. Zweitens wäre zu 
fragen, ob moderne Gesellschaft a priori vor solchen Implosionsprozessen gefeit sind, man also mit absoluter Sicherheit ausschließen kann, daß ihnen Gleiches widerfährt. Wer meint, ein solcher Gedanke wäre absurd, muß sich fragen lassen, ob er nicht den vor unseren Augen ablaufenden Implosionsprozeß noch vor einem Jahr für ebenso absurd gehalten hätte.

Sicher, mit Antworten ist man oft schnell bei der Hand. In den verschiedenen Argumentationsmustern tauchen immer wieder - direkt oder indirekt - zwei Kategorien auf, die besonders geeignet erscheinen, überzeugende Antworten zu konstruieren: Innovation und Stagnation. Den unterschiedlichen Wissens-Kulturen gelten gemeinhin diese beiden Termini als unvereinbare, einander ausschließende Gegensätze. Dementsprechend bipolar sind die damit assoziierten Begriffspaare: Fortschritt/Stillstand, Revolution/Restauration, Kreativität/Routine, Phantasie/Bürokratie, dynamisch/statisch, diskontinuierlich/kontinuierlich, neu/alt, modern/antiquiert usw. Die sich überlagernden Spannungsfelder zwischen diesen verschiedenen Polen bilden ein unerschöpfliches Quellgebiet für Legenden. Wieder und wieder erzählen sich die Menschen Geschichten über den unversöhnlichen Kampf zwischen Innovation und Stagnation. Traurige und euphorische Geschichten, je nachdem, ob man den Sieg der Innovation über die Stagnation beklagt und feiert.

Die Legenden sind Legion.

Diese Legenden eignen sich in besonderer Weise für einfache Antwortschemata. Der »real existierende Sozialismus « implodierte, weil er stagnativ war, die moderne bürgerliche Gesellschaft modernisiert, weil sie innovativ ist - so der rohe Kern der Botschaft. Verhielte es sich so, dann wären die Antworten auf die zuvor gestellten zwei Fragen klar. Erstens, um weiter Implosionsprozesse in Osteuropa zu verhindern bzw. zu verzögern, müssen Innovationspotentiale gestärkt und Stagnationsstrukturen beseitigt werden. Wie dies geschieht, zeigen moderne Gesellschaften. Zweitens, da Implosionsprozesse aus Stagnation resultieren, die Modernisierung moderner Gesellschaften hingegen auf Innovation beruht, bleibt ihnen ein Implosionsschicksal per definitionem erspart.

Verhält es sich so?

Bedenken scheinen immerhin angebracht, ist es doch fraglich, ob sich Implosionsund Modernisierungsprozeß über den argumentativen Mechanismus »Stagnation contra Innovation « so einfach scheiden lassen. Die DDR als eines der entwickeltsten Länder des »real existierenden Sozialismus « zeichnete sich nämlich nicht einfach durch die pure Abwesenheit jeglicher Innovationen aus, sondern vielmehr durch einen spezifischen Innovationspfad, auf dem diese - spärlich genug - zustande kamen. Die äußeren Konturen und inneren Strukturen dieses Pfades könnten aus systematischer Sicht, wenn auch sehr grob, wie folgt beschrieben:

Erstens, die äußeren Konturen. Sie ließen sich stichpunktartig wie folgt skizzieren:

1. Innovationsvoraussetzungen:

a)notwendige Voraussetzungen:

1. globale Systemstabilität

2. starke Systemgrenzen

b) hinreichende Voraussetzungen: lokale Instabilitäten 


\section{Innovationsimpuls:}

3. Innovationsrichtung:

4. Innovationsresultat: lokale und/oder globale Systemstabilisierung

Konsensbildung durch:

\section{Konflikt-Externalisierung}

2.Vetopotential-Minimierung

1. objektive Verflechtung der Handlungsnetze

2. subjektive Entflechtung der Handlungsnetze

Zweitens, die inneren Strukturen. Sie ließen sich stichpunktartig wie folgt skizzieren: Die globale Systemstabilität als die erste notwendige Innovationsvoraussetzung wurde über die Universalierung des Macht-Dispositivs der Partei (Marz 1990e), insbesondere die Theorie des »wahren Wissens«, die »administrative Hand « (Marz 1990b) und deren Internalisierung (Marz 1990d) erreicht. Die starken Systemgrenzen als die zweite notwendige Innovationsvoraussetzung wurden nicht nur durch »die Mauer «, sondern durch eine Vielzahl von Mauern (Autarkieparadigma, Verhinderung eines allgemeinen Zugangs zur Weltkunst und Weltwissenschaft etc.) in allen Bereichen der Gesellschaft gesichert. Die lokalen Instabilitäten als hinreichende Innovationsvoraussetzung waren vergleichsweise sehr schwach ausgeprägt, da das Konzept einer statischen Systemstabilität dominierte, der jede lokale Instabilität automatisch als globale Systemgefährdung galt. Lokale Instabilitäten unterlagen einer doppelten zentralen Kontrolle. Entweder wurden sie ganz bewußt und zielgerichtet erzeugt, oder wenn sie als unbeabsichtigte Nebeneffekte auftraten, energisch bekämpft. Der unmittelbare Innovationsimpuls, bestand zunächst darin, die aufgetretene lokale Instabilität zu beseitigen, und zwar so, daß sich das alte Gefüge der lokalen Handlungskonstellationen (erweitert) reproduziert. Das Minimalziel bestand in der Sicherung, das Maximalziel im Ausbau der Position. Stets war jedoch in allen dementsprechenden Aktivitäten auch die Vorstellung entscheidungs- und handlungsleitend, damit zugleich einen konkreten Beitrag zur globalen Systemstabilisierung zu leisten. Die Innovationsrichtung bestand primär in einer Konsensbildung. Diese wurde auf zwei komplementären Wegen erreicht. Der erste Weg war die Konflikt-Externalisierung. Konflikte wurden aus dem Raum, in dem sie entstanden, exportiert. Hierbei bediente man sich eines verzweigten Systems von Externalisierungswegen. Konflikte wurden aus dem System (zum »Klassenfeind «), in andere Bereich des Systems (von den Medien in die Kunst, insbesondere die Literatur) (Kühne 1975), in die Umwelt (Braunkohle, Luft- und Wasserverschmutzung), in die Zukunft (Senkung der Akkumulations- und Erhöhung der Konsumrate) oder in die Individuen (Überindividualisierung) (Engler 1989a, S. 752) exportiert. Der zweite Weg der Konsensbildung bestand in der Vetopotential-Minimierung. All jenen, die als tatsächlich oder vermeintlich Betroffene einer Innovation gegen diese hätten ein Veto einlegen können, wurden die Möglichkeiten dazu entzogen. Dies geschah nicht nur durch offenen Zwang (Ausbürgerung, Demonstrations-, Organisations- und Medienverbot), sondern auch und vor 
allem durch den stummen, strukturellen Zwang der Verhältnisse. Individuelles und kollektives Vetopotential wurde atomisiert und in eine alltagsweltliche soziale Ohnmächtigkeit gedrängt. Der skizzierte Mechanismus zeitigte zwei entscheidende Innovationsresultate, die unter den Händen der Gesellschaftsmitglieder und hinter ihrem Rücken zu einem stabilen und zunehmend eskalierenden Zwangskreislauf auskristallisierten. Erstens führte die permanente spontane Konflikt-Externalisierung zu einer zunehmenden objektiven Verflechtung der Handlungsnetze, in die die Menschen eingebunden waren und die sie durch ihr tagtägliches Verhalten reproduzierten. Es entstanden immer mehr gordische Knoten, die sich lokal nicht lösen ließen, ohne das gesamte System bis in die Grundfesten zu erschüttern und global zu destabilisieren. Diese Knoten nahmen in solchen Stichworten wie Preis- und Subventionspolitik, Medienpolitik, organisierte Verantwortungslosigkeit, Arbeitsverhalten, Befehls- und Mangelwirtschaft, Umweltschutz, Reisefreiheit usw. zunehmend handgreifliche Gestalt an. Wer lokal an einem dieser Knoten zog, rüttelte, ob er wollte oder nicht, am gesamten System. Zweitens führte die Vetopotential-Minimierung zu einer subjektiven Entflechtung der Handlungsnetze. Im wachsenden Maße traten an den verschiedensten Punkten des gesellschaftlichen Handlungsraumes für die Menschen Wahrheit, Richtigkeit und Sinn ihrer Handlungen zunehmend auseinander. Die Menschen waren in ihren verschiedenen Alltagswelten in gegensätzliche (und teilweise obskure) ${ }^{9}$ Handlungsrationalitäten eingebunden, denen sie zwar formell entsprachen, die sie jedoch individuell immer weniger vermitteln konnten. Man nahm an Versammlungen teil und wußte zugleich, daß es vertane Zeit ist, da sich eh nichts ändern würde, und dies nicht so sehr, weil die anwesenden Entscheidungsträger nichts ändern wollten, sondern weil man nur zu oft wußte, daß sie nichts ändern konnten; man nahm an den diversen Initiativbewegungen teil, obwohl man es persönlich für sinnlos hielt und jeder die Wahrheit der medialen Berichterstattungen über ihre gesamtgesellschaftlichen Resultate bezweifelte; man drosch selbst dann noch Phrasen, wenn keiner es hören wollte, weil es allemal bequemer war, fün absurde Argumentationen abzuspulen, als einen eigenen Gedanken zu fassen, der ohnehin nichts bewirken würde. Auf die subjektive Entflechtung der Handlungsnetze reagierten die Menschen mit schizophren anmutenden Verhaltensmustern und inner Immigration. Beide Innovationsresultate, objektive Ver- und subjektive Entflechtung der Handlungsnetze untergruben eine der notwendigen Innovationsvoraussetzungen, die globale Systemstabilität. Der Innovationspfad war rekursiv geschlossen, der Prozeß begann von vorn.

In dem beschriebenen Mechanismus standen sich zwar punktuell Innovation und Stagnation gegenüber, das Typische war jedoch die Verflechtung und Verschmelzung beider, zu einem Zwangskreislauf, der sich vielleicht am besten als Stagnovation bezeichnen ließe. Es wurde lokal innoviert, aber so, daß tendenziell eine globale Stagnation eintrat. Jede Innovation wurde kolonisiert und diente in dieser oder jener Form derZementierung der Stagnation. Die Einführung der Computertechnik führte primär zu einer C-technisch gestïtzten Stabilisierung der »administrativen Hand « (Marz 1990 b, S. 66 f.), machtkritische Literatur nährte das »wahre Wissen«, lokale wissenschaftliche, technische oder sportliche Spitzenleistungen galten als Ausweis der glo- 
balen Systemstärke. Das Gefängnis der Stagnovation erhielt die Aura sozialer Geborgenheit. Es war eine statische Stagnovation.

Wenn nun der Implosionsprozeß einer »real sozialistischen« Gesellschaft nicht schlechthin aus einer universellen Stagnation, sondern vielmehr aus einer spezifischen Verschmelzung von Stagnation und Innovation, eben der statischen Stagnovation resultierte, dann könnte gefragt werden, ob es sich hierbei nur um ein »real sozialistisches « Phänomen handelt. Natürlich, es gab zweifellos spezifische Elemente, etwa die Art und Weise, in der die globale Systemstabilisierung sichergestellt wurde oder die speziellen Formen, in denen die Konsensbildung erfolgte. Dennoch wäre es vielleicht etwas voreilig, die Frage nach der Bedeutsamkeit des Stagnovationśproblems für die Modernisierung moderner Gesellschaften ohne weitere Umschweife abschlägig zu beantworten. Freilich, statische Stagnovationskreisläufe dürften kaum oder gar nicht auszumachen sein. Zu prüfen bliebe indes, ob nicht Tendenzen einer dynamischen Stagnovation festzustellen sind. Konflikt-Externalisierung, Vetopotential-Minimierung, objektive Ver- und subjektive Entflechtung der Handlungsnetze usw. sind Phänomene, die sich - wenn auch in völlig anderen Formen - auch im Modernisierungsprozeß moderner Gesellschaften unschwer auffinden lassen dürften. Die Frage, ob sie sich zu einem eskalierenden Zwangskreislauf verdichten könnten, sollte auch von jenen diskutiert werden, die eine dynamische Stagnovation weder gegenwärtig sehen noch prinzipiell für möglich halten. Zu viele haben zu lange zuviel für unmöglich gehalten.

\section{Anmerkungen}

1 Die Konstruktion dieses exklusiven Punktes oblag dem sogenannten »M/L«. Wie diese, unter dem Namen »Marxismus-Leninismus« firmierende Ordnungswissenschaft, diese Aufgabe bewältigte vgl. Krüger 1990a; Marz 1990c, S. 57.

2 Die Formen in denen dies geschieht, sind ebenso vielgestaltig wie diffizil. Erinnert sei hier nur an Koestler 1973; Lodge 1984; Bourdieu 1988; Dreyfus, St. E. 1986; Luhmann 1986, Dreyfus/Rabinow 1987.

3 Zu den arabesken Formen, die die Paradoxien der Selbstreferenz annehmen können vgl. Hofstadter 1986; Hofstadter 1988.

4 Gegenwärtig läßt sich dieses Phänomen am Niedergang der Wissens-Kultur des »wahren Wissens« beobachten. Vgl. dazu Marz 1990e.

5 Ebenso augenfällig wie folgenreich ist diese Ohnmacht in der Wirtschaft. Dazu vgl. Marz 1990d.

6 Bei der Beschreibung des Innovationspfades - insbesondere was die Bedeutung der Konsensbildung (Konflikt-Externalisierung und Vetopotential-Minimierung) für Innovationsprozesse betrifft - verdanke ich Jeanette Hofman vom WZB/SP II OT viele methodologische Anregungen, Ideen und kritische Diskussion.

7 Besonders auffällig war dies in der Wirtschaft. Hier wurdèn einerseits mit einem unvorstellbarem administrativen, personellen, finanziellen und ideologischen Aufwand die Leitungsstrukturen der Kombinate und Werke über ein Befehls-, Melde- und Kontrollsystem unter Druck gesetzt, um Innovation Raum zu schaffen (»Mikroelektronikprogramm«, »Neuererwesen«, »Pflichtenhefte« etc.), andérerseits beschäftigten sich die verschiedensten Organe der »administrativen Hand« (Politbüro, Ministerrat, Ministerien, Bezirks- und Kreisleitungen der SED etc.) permanent mit der Beseitigung von ver- 
sorgungsengpässen. Nicht gerade selten konnte eine Mangelware (Zahnbürsten, Kaffee, Verbandsmaterial, Joghurt, Ketchup, Rosinen, Bettwäsche, Warmgeräteanschlußleitungen, Babykostwärmer etc.) hunderte Kader monatelang in Atem halten.

8 Solche Losungen etwa wie »Mein Arbeitsplatz ist ein Kampfplatz für den Frieden«, »Plane mit, arbeite mit, regiere mit « oder »Je stärker der Sozialismus, desto sicherer der Friede« waren mehrals bloße »von oben« verordnete Propagandahülsen. Diese und ähnliche Schlagworte brachten das eingangs skizzierte Gefuihl auf den Begriff, in einer überlegenen Alternativgesellschaft zu leben. Die alltagsweltlich ebenso handlungsleitende wie -mobilisierende Bedeutsamkeit dieser massenhaft verbreiteten Vorstellung wurde und wird nun zunehmend unterschätzt. Vielen ist es jetzt peinlich, sich in diese Situation zurückzufühlen. Es fällt leichter, die Geschichte als eine Geschichte diverser äußerer Druck- und Zwangssysteme zu erinnern, denen man leidend ausgesetzt war, als danach zu fragen, warum und wie die psychischen Selbstzwänge oft stärker werden konnten als die sozialen Fremdzwänge und inwiefern ihnen auch bestimmte Momente des erhabenen Genusses inne waren. Dazu vgl. auch Marz 1990c; Marz 1990d.

9 Eine solche obskure Handlungsrationalität war z.B. das sogenannte Melde(un)wesen in der Wirtschaft. Für einen Beobachter mag folgendes Phänomen nicht nur absurd, sondern schlicht unglaublich erscheinen: Ein Betrieb überbot in einer Erzeugnisnomenklatur seinen Monatsplan. Am 2. Arbeitstag des neuen Monats wurde ihm in dieser Position rückwirkend der Plan für den Vormonat so erhöht, daß er vom »Übererfüller« zum »Untererfüller« wurde. Gleichzeitig wurde er beauflagt, diese »Untererfüllung « exakt zu begründen und eine »Abbaukonzeption « für die Planrückstände zu erarbeiten und einzureichen. Dies galt für die daran Beteiligten zwar ats belastendes, nichtsdestoweniger jedoch $\gg$ Normale«, weil handlungsrational logische Arbeitsaufgabe, der man sich - wenn auch vielleicht zähneknirschend - stellte. Es verstand sich von selbst, daß als Grund für die »Planuntererfüllung « nicht die rückwirkende Planerhöhung, sondern vielmehr »objektive und subjektive« Probleme im eigenen Verantwortungsbereich zu benennen waren, für deren umgehende Beseitigung man sofort alle notwendigen Maßnahmen einleitete.

\section{Literatur}

Bourdieu, P. (1988): Homo academicus. Frankfurt/a.M.

Bühler, K. (1965): Sprachtheorie. Die Darstellungsfunktion der Sprache. Stuttgart

Dreyfus, H.L./Dreyfus, St.E. (1986): Mind over Machine. The Power of Human Intuition and Expertise in the Era of the Computer. New York

Dreyfus, H.L./Rabinow, P. (1987): Michel Foucault. Jenseits von Strukturalismus und Hermeneutik. Frankfurt/a.M.

Elias, N. (1985): Die höfische Gesellschaft. Untersuchungen zur Soziologie des Königtums und der höfischen Aristokratie. Mit einer Einleitung: Soziologie und Geschichtswissenschaft. Frankfurt/a.M.

Engler, W. (1989a): Gespräch mit Norbert Elias. In: Sinn und Form, Heft 4/1989. S. 743 ff.

Ders. (1989b): Die kleinen Erzählungen Universalisten und Kontextualisten im Streit um die Grundlagen der Menschenwissenschaften. In: Zeitschrift für Germanistik (Leipzig), Nr. 5/1989, S. 537 ff.

Ders. (1989c): Teilnehmen und Beobachten. Zur Kritik der Wissenssoziologie. (Dissertation B am Institut für Theorie, Geschichte und Organisation der Wissenschaften der Akademie der Wissenschaften der DDR). Berlin

Ders. (1990): Wissensproduktion. Über (Un)möglichkeiten kritischer Forschung. In: Kommune, Nr. 3/ 1990. S. $68 \mathrm{ff}$.

Friese, H./Wagner, P. (1990): Die Revolution und das Ende der Utopie. Über die Domestizierung der Wirklichkeit und den Beginn der Rückeroberung. In: Stachlige Argumente, Nr. 62 (Mai 1990). S. 47 ff.

Gödel, K. (1931): Über formal unentscheidbare Sätze der Principia Mathematica und verwandter Systeme. In: Monatshefte für Mathematik und Physik, S. $173 \mathrm{ff}$.

Habermas, J. (1981): Theorie des kommunikativen Handelns. Bd. 2, Frankfurt a.M.

Hofstadter, D.R. (1986): Gödel, Escher, Bach. Ein endlos geflochtenes Band. Stuttgart 
Ders. (1988): Metamagicum. Fragen nach der Essenz von Geist und Struktur, Stuttgart

Koestler, A. (1973): Die Herren Call-Girls. München

Krüger, H.-P. (1988): Kommunikationstheoretische Fragen der Wissenschaftsentwicklung. In: Kröber, G. (Hrsg.): Wissenschaft Das Problem ihrer Entwicklung. Bd. 2. Komplementäre Studien zur marxistischIeninistischen Wissenschaftstheorie. Berlin. S. $113 \mathrm{ff}$.

Ders. (1989): Produktion und Kommunikation oder Marx und Habermas, In: Sinn und Form, Heft 6/1989. S. $1183 \mathrm{ff}$.

Ders. (1990a): Moderne Gesellschaft und »Marxismus-Leninismus« schließen einander aus. In: Initial, Heft 2/1990. S. 149 ff.

Ders. (1990b): Zur Differenz zwischen kapitalistischer und modemer Gesellschaft. In: Deutsche Zeitschrift für Philosophie, Heft 3/1990. S. $202 \mathrm{ff}$.

Kühne, L. (1975): Literatur und Ideologie. Über den Zusammenhang kunstliterarischer und theoretischer Aneignung der Wirklichkeit. In: Herausgeberkollektiv: Funktion der Literatur. Aspekte - Probleme Aufgaben. Reihe: Literatur und Gesellschaft. Berlin. S. 340 ff.

Lodge, D. (1984): Small World. An Academic Romance. London

Luhmann, N. (1986): Ökologische Kommunikation. Kann die moderne Gesellschaft sich auf ökologische Gefährdungen einstellen? Opladen

Marz, L. (1990a): Zu einer modemen Ökonomie der DDR. Leitlinien für eine öffentliche Strategiediskussion. In: PROKLA, Nr. 78, S. 13 ff.

Ders. (1990b): Die Ohnmacht der Altmacht. Zur Anatomie der administrativen Hand. In: Kommune, Nr. 3/1990.S. $63 \mathrm{ff}$.

Ders. (1990c): Illusionen und Visionen. Leitbilder von und in modernen Gesellschaften,. In: Kommune, Nr. 6/1990. S. $55 \mathrm{ff}$.

Ders. (1990d): Der prämoderne Übergangsmanager. Die Ohnmacht des »real sozialistischen« Wirtschaftskaders. (erscheint im November 1990 in einem Sammelband bei Suhrkamp)

Ders. (1990e): Das Macht-Dispositiv der Partei. Das Régime des Wissens im »real existierenden Sozialismus«. (erscheint in: Deutsche Zeitschrift für Philosophie, Heft 10/1990)

Maturana, H.R./Varela, F.J. (1987): Der Baum der Erkenntnis. Wie wir die Welt durch unsere Wahrnehmung erschaffen - die biologischen Wurzeln des menschlichen Erkennens. Bern/München/Wiesbaden 